\title{
Bilateral Metastatic Renal Hemangiopericytoma Ten Years after Primary Intracranial Lesion
}

\author{
Lee N. Hammontree, Kristopher Whitehead, James M. Markert \\ Department of Surgery, Divisions of Urology and Neurosurgery, University of Alabama at Birmingham, \\ Birmingham, Alabama, USA
}

\begin{abstract}
We report a case of bilateral metastatic renal hemangiopericytoma. A 37-year-old Caucasian male presented in 1993 with intracranial hemangiopericytoma. Subsequent metastatic disease noted years later include bilateral renal hemangiopericytoma 10 years after initial presentation. To our knowledge, this is only the second reported case of bilateral metastatic renal hemangiopericytoma.
\end{abstract}

Key words: intracranial neoplasms; hemangiopericytoma; kidney; metastases; bilateral Int Braz J Urol. 2006; 32: 306-7

\section{INTRODUCTION}

Since Black \& Heinemann are credited as first described a renal hemangiopericytoma in 1955 , 34 other cases have been reported. Only one report described bilateral renal involvement. This rarity occurred in 1991 when Heppe et al. described a male patient with bilateral metastases 12 years after an initial intracranial lesion (1). To our knowledge, this is only the second reported case of bilateral metastatic renal hemangiopericytoma.

\section{CASE REPORT}

A 37-year-old white male presented in 1993 with headaches and dizziness, vomiting, and personality changes. A large dural-based middle fossa intracranial mass was discovered and excised after a subdural hematoma developed following embolization.
Cellular morphology was consistent with hemangiopericytoma. Subsequently, 2 episodes of recurrence within the middle fossa were noted on follow-up imaging. The patient's intracranial lesions responded favorably to gamma knife irradiation and further imaging studies were done to detect metastatic lesions. Abdominal imaging demonstrated 2 large enhancing lesions in the right hepatic lobe. After resection, examination of the specimen revealed 2 large foci $(13.5 \mathrm{~cm}$ and $10.5 \mathrm{~cm})$ of metastatic disease. Immunoperoxidase staining for cytokeratin, EMA, actin, and CD-34 markers showed only CD-34 stain uptake in the prominent vascularity of the lesions.

These radiographic studies also revealed a 1.5 $\mathrm{x} 1.3 \mathrm{~cm}$ isodense mass arising from the lower pole of the left kidney. Growth occurred over time. A new $4 \mathrm{~mm}$ mass of unknown significance also developed during this interval in the mid to lower pole of the right kidney. Plans were made to remove the left kidney after no further evidence of metastasis was 
noted on PET scan. In December 2003, a left handassisted laparoscopic radical nephrectomy was performed. Pathologic examination confirmed a 2.4 x $2.3 \times 2.3 \mathrm{~cm}$ hemangiopericytoma with focal areas of hemorrhage. A smaller focus of tumor with similar characteristics was also found in the specimen. Both metastases were confined within the boundaries of the renal capsule.

In May 2004, many lesions appeared in the lateral segment of the left hepatic lobe and pancreatic body. Interval enlargement was also noted in the right renal mass, which had increased in size to $2.7 \times 1.1$ $\mathrm{cm}$. The aggressive nature already demonstrated by this malignancy, presence of multiple metastases, and similar progression of the contralateral renal mass are evidence to conclude bilateral renal involvement.

\section{COMMENT}

Grossly, these tumors do not share a characteristic appearance but do tend to grow rapidly within the confinements of a capsule or pseudocapsule, but calcifications are seldom evident unless tumors have been present for long durations. Histological confirmation also is difficult because of microscopic similarities that exist between these lesions and other soft tissue tumors, namely synovial sarcomas, fibrous histiocytomas, and solitary fibrous tumors. It should be noted that while most hemangiopericytomas express CD-34 as illustrated by our case, the sensitivity of this marker is suboptimal in some cases, often limited to endothelial cells (2).
Despite reports of hemangiopericytomas in all age ranges, 2 distinct clinical types with separate prognoses have emerged. An infantile form exists only during the first year of life, while over $90 \%$ occur during adulthood with a peak incidence in the fifth and sixth decades. Efforts to correlate such long-term outcomes with factors such as tumor size, degree of tumor necrosis, and presence of mitotic figures have only achieved limited success. The single prognostic indicator after primary diagnosis remains the presence or absence of metastasis. Studies have shown the most important factor in reducing tumor spread to be complete excision of the primary lesion (3).

\section{CONFLICT OF INTEREST}

None declared.

\section{REFERENCES}

1. Heppe RK, Donohue RE, Clark JE: Bilateral renal hemangiopericytoma. Urology. 1991; 38: 249-53.

2. Cohen PR, Rapini RP, Farhood AI: Expression of the human hematopoietic progenitor cell antigen CD34 in vascular and spindle cell tumors. J Cutan Pathol. 1993; 20: $15-20$.

3. Guthrie BL, Ebersold MJ, Scheithauer BW, Shaw EG: Meningeal hemangiopericytoma: histopathological features, treatment, and long-term follow-up of 44 cases. Neurosurgery. 1989; 25: 514-22.

\author{
Correspondence address: \\ Dr. Lee N. Hammontree \\ FOT 1105 \\ 1530 3rd Ave S \\ Birmingham, AL, 35294-3411, USA \\ Fax: + 1205 934-4933 \\ E-mail: leehammontree@ccc.uab.edu
}

